\title{
Pencils of curves on smooth surfaces
}

\author{
A. Melle-Hernández and C.T.C. Wall
}

June 28, 2000

In the study of polynomial functions of two variables over the complex numbers, it has been known from the beginning (see e.g. [4]) that the family was topologically trivial except at a finite number of atypical values, and an early and striking theorem [16] asserts that these values are characterised by a simple Euler characteristic condition. The starting point of this work was an attempt to see what became of this result for curves in finite characteristic.

To understand the geometry one is soon led to compactify the family by adding in points at infinity, and it then seems more natural to consider arbitrary pencils of curves. Since one is soon led to perform blowing up, there is little to be gained by restricting to families on a plane, so this can be replaced by an arbitrary smooth projective surface.

Moreover, to direct the study in finite characteristic it is essential to begin with a clear picture of the situation in characteristic zero. Here the paper [20] of Lê and Weber shows that the characterisation of atypical fibres by Euler characteristics applies locally. However their purely topological reasoning cannot be used in finite characteristic, and we need to replace it by algebraic arguments.

In finite characteristic there are two essential changes to the situation in characteristic zero. One is the existence of 'wild vanishing cycles', enumerated by the 'Swan conductor', which measures the failure of the familiar behaviour of the Euler characteristic for fibrations. The other is the failure of Bertini's theorem. Although in some sense this is now well understood, the geometrical picture is significantly changed when a failure presents itself, and we do not at present have a satisfactory account of these situations.

We begin with a preliminary section introducing our main tools and concepts. We recall a number of known results on maps from a smooth surface to a smooth curve with smooth general fibre: here the Swan conductor enters the formulae, but does not really affect the condition for a fibre to be typical. In $\S 3$ we give a brief discussion of general pencils, and in the final section we present a characterisation of exceptional members of a pencil by Euler characteristics, assuming a 'conservative' condition equivalent to Bertini's theorem.

Let $k$ be a field of characteristic $p \geq 0$. Let $X$ be a surface over $k$. A point $x \in X$ is smooth if and only if the corresponding points $x^{\prime}$ over algebraically 
closed $k^{\prime} \supset k$ are smooth. If $k$ is algebraically closed, or more generally if $k$ is perfect, this is equivalent to $x$ being a regular point of $X$. In general, smooth implies regular but not conversely: we return to this below. A point $x \in X$ is singular if it is not smooth. If $k$ is an algebraically closed field then $X$ is smooth at all points if $X$ is a non-singular surface, e.g. see [17].

Most of this work was done while the first author was a visitor at the University of Liverpool. The kind hospitality of the Department of Mathematical Sciences at Liverpool is gratefully acknowledged. The research of the first author was partially supported by a grant 'Convenio SEUID - Royal Society' and by DGES PB97-0284-CO2.

\section{Preliminaries}

We begin by recalling a number of known results, in which the behaviour of curves in characteristic $p$ is parallel to that in the classical case. Here and below, 'curve' means a scheme of dimension one of finite type over $k$.

\subsection{Resolution of singularities of a curve}

Singularities of curves in finite characteristic may be resolved, as in the classical case, by blowing up points: see e.g. [6]. We recall some properties of this procedure, which survive in positive characteristic [8]. We begin with a reduced curve $C$ lying in a smooth surface $X$.

A single step consists in choosing a singular point $x$ of $C$ and blowing $X$ up at this point. This gives a smooth surface $\tilde{X}$ and a map $\pi: \tilde{X} \rightarrow X$. The surface $\tilde{X}$ contains an exceptional curve $E=\pi^{-1}(x)$, and the strict transform $\tilde{C}$ of $C$, which may be found as the closure of $\pi^{-1}(C)-E$. When we consider $C$ as a divisor, we have the total transform $\pi^{*}(C)$ and the strict transform $\pi^{*}(C)-m_{x}(C) E$, which does not contain $E$ as a component. Here $m_{x}(C)$ is the multiplicity of $C$ at $x$. The exceptional curve $E$ is smooth and rational, and we have $\tilde{C} \cdot E=m_{x}(C)$.

Any sequence of blowings up yields a sequence of surfaces, which contain, as well as strict transforms of the original curve $C$, the strict transforms of the successive exceptional curves. In each of these surfaces, the collection of all exceptional curves lying over $x \in X$ forms a tree of curves, with normal crossings.

We distinguish the exceptional curves by suffices, and e.g. let $E_{\alpha}$ be created by blowing up a point $e_{\alpha}$. We continue to write $E_{\alpha}$ for the strict transforms of $E_{\alpha}$ in all higher surfaces. In the terminology of Enriques, each $E_{\alpha}$ determines an infinitely near point to its image $x \in X$.

The sequence of blowings up induces a partial order on the infinitely near points $e_{\alpha}$ : we say $e_{\beta}$ is proximate to $e_{\alpha}$ if the point $e_{\beta}$ lies on the curve $E_{\alpha}$, and that $e_{\beta}$ lies above $e_{\alpha}$ if there is a sequence of points $e_{\beta}, e_{\gamma}, \ldots, e_{\alpha}$ with each one proximate to the next. 
Let $X$ be a smooth projective surface defined over $k$, let $x$ be a closed point on $X$ and $X^{*}$ the set of infinitely near points of $x$. For $D$ an effective divisor on $X$ and $p \in X^{*}$, denote by $m_{p}(D)$ the multiplicity of the strict transform of $D$ at $p$. If $E$ is another effective divisor on $X$ such that $D$ and $E$ intersect at $x$ and have no common components through $x$, then the intersection multiplicity of $D$ and $E$ at $x$ is given by

$$
(D . E)_{x}=\sum_{p \in X^{*}} m_{p}(D) m_{p}(E)[k(p): k],
$$

where the sum is over the infinitely near points $p$ of $x$ in common to $D$ and $E$ and $k(p)$ is the residue field at the point $p$. If $k$ is algebraically closed, then each infinitely near point $p$ is rational over $k$, so the integer $[k(p): k]$ is equal to 1 .

If $D$ is a reduced curve on $X$ with $x \in D$, then the 'double point number' $\delta_{x}(D)$ is given by

$$
\delta_{x}(D)=\frac{1}{2} \sum_{p \in X^{*}} m_{p}(D)\left(m_{p}(D)-1\right)[k(p): k],
$$

and we have the proximity relations

$$
m_{p}(D)=\sum_{p^{\prime}} m_{p^{\prime}}(D)\left[k\left(p^{\prime}\right): k(p)\right]
$$

where the sum is over all the infinitely near points $p^{\prime}$ proximate to $p$.

Lemma 1.1 Let $C$ be an irreducible curve germ over an algebraically closed field; suppose repeatedly blowing up $C$ produces the sequence $\left\{p_{i}: i \geq 0\right\}$ of infinitely near points, and that $p_{n+2}$ is not proximate to any $p_{i}$ with $i<n$. Then there are numbers $a, b$, determined solely by the proximity relations, such that for any curve $\Gamma$ through $p_{n+1}$,

$$
m_{0}(\Gamma)=a m_{n}(\Gamma)+b m_{n+1}(\Gamma)
$$

Proof Suppose inductively we have found $a_{r}, b_{r}$ such that for all $\Gamma$ through $p_{n+1}$ we have $m_{n-r}(\Gamma)=a_{r} m_{n}(\Gamma)+b_{r} m_{n+1}(\Gamma)$ - the induction starts trivially with $a_{-1}=0, b_{-1}=1, a_{0}=1, b_{0}=0$. Then the multiplicity at $p_{n-r-1}$ is the sum of the multiplicities at all points proximate to that one, which must be $p_{n-r}, \ldots, p_{n-s}$ where $s \geq-1$ since it follows from our hypothesis that no $p_{q}$ with $q>n+1$ can be proximate to any $p_{i}$ with $i<n$. Thus we may take $a_{r+1}=\sum_{s}^{r} a_{i}$ and similarly for $b_{r+1}$.

\subsection{Resolving base points of pencils of curves}

We now collect some well-known results on resolutions of pencils on a smooth projective surface $X$ over an algebraically closed field $k$. Let $\Lambda$ be a pencil of curves $\left\{\Gamma_{t}\right\}$ on $X$ which has no fixed component, and let $x \in X$ be any point of intersection of two, hence of all curves in the pencil: such points are called base 
points. Write $m_{x}(\Lambda)=\min \left\{m_{x}\left(\Gamma_{t}\right) \mid t \in P^{1}\right\}$. Then $m_{x}(\Lambda)=m_{x}\left(\Gamma_{t}\right)$ for all but finitely many $t \in P^{1}$.

Let $\pi: \tilde{X} \rightarrow X$ be the blow up of $X$ at $x, E$ the exceptional divisor of $\pi$. Then the total transform $\pi^{*}(\Lambda)=\left\{\pi^{*}\left(\Gamma_{t}\right) \mid \Gamma_{t} \in \Lambda\right\}$ of $\Lambda$ is a linear system with $m_{x}(\Lambda) E$ as fixed component. Removing this gives the strict transform $\Lambda_{1}=$ $\left\{\pi^{*}\left(\Gamma_{t}\right)-m_{x}(\Lambda) E \mid t \in P^{1}\right\}$ of $\Lambda$, which has no fixed component.

Let $q \in X_{r}$ be an infinitely near point of $p$ obtained by a finite sequence of blowings-up $\pi_{i}: X_{i+1} \rightarrow X_{i}$ (with $\left.X_{0}=X\right)$. Set $m_{q}(\Lambda):=m_{q}\left(\Lambda_{r}\right)$ where $\Lambda_{r}$ is the strict transform of $\Lambda$ on $X_{r}$. We say that $q$ is a base point of $\Lambda$ if $m_{q}(\Lambda)>0$.

Inductively blow up at a base point of the pencil, take the strict transform of the pencil, and continue. Since for two members of the pencil with multiplicity $m_{x}(\Lambda)$ at $x$, blowing up $x$ reduces their intersection multiplicity there by $m_{x}(\Lambda)^{2}$, the blow-up reduces the total intersection number of these two, hence of any two members of the pencil by $m_{x}(\Lambda)^{2}$. As the original intersection number is finite, we may continue till no base points remain. Write $B$ for the set of base points (including infinitely near ones) of $\Lambda$ : then this is a finite set.

At the end, we have a smooth projective surface $Y$ with a well defined map $\pi: Y \rightarrow P^{1}$, whose fibres $Y_{t}=\pi^{-1}(t)$ project to the curves $\Gamma_{t}$ of the original pencil. In local coordinates $(u, v)$ on $Y$ we may write $\pi(u, v)=(f(u, v): g(u, v))$ where $f, g$ are algebraic functions on $Y$, smooth at the point in question; then $Y_{t}$ is given by $f=t g$.

An exceptional curve in $Y$ may project to a single point of $P^{1}$ and so lie in a fibre, when we call it vertical, or may map onto $P^{1}$, in which case we call it horizontal or dicritical.

We now discuss the creation of a single exceptional curve $E_{\alpha}$ : write $m=$ $m_{\alpha}:=m_{e_{\alpha}}(\Lambda)$. Take local coordinates in $X_{\alpha}$ with $e_{\alpha}$ at $(0,0)$. Then we can write $f=\sum_{m}^{\infty} f_{k}$ as a sum of homogeneous components, and similarly for $g$. The $m$-jets $f_{m}, g_{m}$ define a pencil of binary $m$-ics. The curve $E_{\alpha}$ has parameters $(\xi: \eta)=(u: v)$ whose projection to the base curve is given by $f_{m}=t g_{m}$. Thus $E_{\alpha}$ is vertical if and only if some member of the pencil of binary forms vanishes identically.

To describe the geometry when $E_{\alpha}$ is horizontal, first note that any common factor of $f_{m}$ and $g_{m}$ produces a base point of the blown-up pencil on $E_{\alpha}$. To determine the multiplicities of these requires an examination of terms of higher order in $f$ and $g$ : for example, $x=0$ defines a singular point of the strict transform in $Y_{\alpha}$ of $f=0$ if and only if $y^{2}$ divides $f_{m}$ and $y$ divides $f_{m+1}$.

If the degree of the highest common factor of $f_{m}$ and $g_{m}$ is $h$ then the degree of the projection $\pi_{\alpha}: E_{\alpha} \rightarrow P^{1}$ is $m-h$. If $f_{m} / g_{m}$ is a function of $(u / v)^{p}$, where $p$ is the characteristic, then all values of $t$ give multiple roots, and to study the geometry it is necessary to factorise $\pi_{\alpha}=\pi_{i} \circ \pi_{s}$ where $\pi_{i}(t)=t^{p^{r}}$ for some $r$, and $\pi_{s}$ is not a function of $(u / v)^{p}$ : here $q=p^{r}$ is called the degree of inseparability. Equivalently, since $k$ is algebraically closed, we may write $f_{m} / g_{m}=$ $\phi^{q}$ and factorise $\pi_{\alpha}=\pi_{\alpha}^{\prime} \circ F_{q}$, where $F_{q}: E_{\alpha} \rightarrow D$ is purely inseparable of degree 
$q$ and $\pi_{\alpha}^{\prime}: D \rightarrow P^{1}$ is separable, of degree $q^{-1}(m-h)$. We will refer to the points of $P^{1}$ over which $\pi_{\alpha}^{\prime}$ is ramified as the branch values of $E_{\alpha}$.

If $q>1$ all points of $E_{\alpha}$ are singular for $\pi_{\alpha}$, and the branching behaviour is best studied via $\pi_{\alpha}^{\prime}$. The number of branch points of $\pi_{\alpha}^{\prime}$ (counting multiplicities appropriately) is $2 q^{-1}(m-h)-2$. The intersections of $E_{\alpha}$ with the fibre $Y_{t}$ are the preimages of $t \in P^{1}$ under $\pi_{\alpha}$ : for almost all $t$ this gives $q^{-1}(m-h)$ distinct points, but for $t$ a branch value of $\pi_{\alpha}^{\prime}$ the number is decreased.

Now suppose $E_{\alpha}$ is vertical: we may suppose $g_{m} \equiv 0$. Let the least order terms in $g$ have degree $m+k$. Then $E_{\alpha}$ lies in the fibre $Y_{s}$ over the point $s$ where $t=\infty$, and has multiplicity $k$ as component of that fibre. The base points of the pencil in the surface $Y_{\alpha}$ are the points on $E_{\alpha}$ where $f_{m}$ vanishes, and $E_{\alpha}$ intersects its complement $Z$ in the corresponding fibre $Y_{s, \alpha}^{\prime}$ of the map $\pi_{\alpha}: Y_{\alpha} \rightarrow P^{1}$ in the points where $g_{m+k}=0$. Although the roots of $g_{m+k}$ need not be distinct, the intersection number of $Z$ and $E_{\alpha}$ is $m+k$.

Lemma 1.2 Any exceptional curve of the first kind contained in a fibre $Y_{t}$ of $Y$ must be a component of $\tilde{\Gamma}_{t}$.

For at the creation of a vertical exceptional curve $E_{\alpha}$ there are at least some branch points on it, so in $Y$ each such curve has been blown up at least once more, so is not exceptional of the first kind.

\subsection{Cohomology and Euler characteristics of curves}

An Euler characteristic is an alternating sum of Betti numbers. In finite characteristic, it is necessary to use étale cohomology groups, which were introduced by Grothendieck [14]. We recall that one first chooses a prime $\ell$ different from $p$, constructs cohomology groups $H_{e t t}^{r}\left(X ; \mathbb{Z} / \ell^{k}\right)$ with finite coefficient groups, and then takes the inverse limit $H_{e ́ t}^{r}\left(X ; \widehat{\mathbb{Z}}_{\ell}\right)$. For our purposes we need only consider the case when $X$ is a proper scheme.

Let $C$ be a reduced, complete algebraic curve over an algebraically closed field $k$. Let $C=\bigcup_{i=1}^{h} C_{i}$ be its decomposition into irreducible components; write also $s$ for the number of connected components of $C$. Let $\bar{C}_{i}$ be the normalisation of $C_{i}, \bar{C}$ be the disjoint union of the $\bar{C}_{i}$ and $n: \bar{C} \rightarrow C$ be the canonical projection. Write $g_{i}$ for the genus of $\bar{C}_{i}$ and $g:=\sum g_{i}$.

For each closed point $x$ of $C$ set $\delta_{x}(C):=\operatorname{dim}_{k} n_{*}\left(\mathcal{O}_{\bar{C}}\right)_{x} / \mathcal{O}_{C, x}$, (see also (1)) and write $\delta(C):=\sum_{x \in|C|} \delta_{x}(C)$ where $x \in|C|$ means that $x$ runs through closed points of $C$. These numbers are finite because $n$ is a finite map and $n_{*}\left(\mathcal{O}_{\bar{C}}\right) / \mathcal{O}_{C}$ is a coherent sheaf supported on a finite set of points. If $r_{x}(C)$ denotes the number of analytic branches of $C$ at $x$ let $\delta_{x}^{\prime}(C):=r_{x}(C)-1$ and $\delta^{\prime}(C):=\sum_{x \in|C|} \delta_{x}^{\prime}(C)$. We use $\mu_{x}(C):=2 \delta_{x}(C)-\delta_{x}^{\prime}(C)$ as a definition of the Milnor number of $C$ at $x$ and write $\mu(C):=\sum_{x \in|C|} \mu_{x}(C)=2 \delta(C)-\delta^{\prime}(C)$ for the total Milnor number of 
$C$. Note that, in distinction to the characteristic zero case, this number cannot easily be calculated from a local equation for $C$ : we discuss this point fully below.

Other invariants of $C$ may now be expressed in terms of the above. Dolgachev [11] showed that the étale cohomology groups of $C$ are free of rank $\beta_{i}(C)$, where the Betti numbers $\beta_{i}(C)$ are given by

$$
\beta_{0}(C)=s ; \quad \beta_{1}(C)=2 g+\delta^{\prime}(C)-h+s ; \quad \beta_{2}(C)=h ; \quad \beta_{i}(C)=0 \text { for } i>0 .
$$

Thus the topological Euler-Poincaré characteristic (l-adic Euler-Poincaré characteristic) of $C$ is

$$
\chi(C)=\chi_{\text {ét }}(C)=\sum(-1)^{i} \beta_{i}(C)=2 h-2 g-\delta^{\prime}(C) .
$$

Thus if $p: C^{\prime} \rightarrow C$ is a birational map (e.g. obtained via blowing up), then the values of $h$ and $g$ agree for $C$ and $C^{\prime}$, so $\chi\left(C^{\prime}\right)-\chi(C)=\delta^{\prime}(C)-\delta^{\prime}\left(C^{\prime}\right)$. This can be written in the more familiar form $\sum_{x \in C} \#\left(p^{-1}(x)-1\right)$.

The cohomology of the sheaf $\mathcal{O}_{C}$ is given in terms of the above invariants (via the Leray spectral sequence for the morphism $n$ ) by:

$$
\operatorname{dim}_{k} H^{0}\left(C, \mathcal{O}_{C}\right)=s, \quad p_{a}(C):=\operatorname{dim}_{k} H^{1}\left(C, \mathcal{O}_{C}\right)=g+\delta(C)-h+s,
$$

so $\chi\left(\mathcal{O}_{C}\right)=h-g-\delta(C)$, and we obtain the useful formula

$$
\chi(C)=2 \chi\left(\mathcal{O}_{C}\right)+\mu(C) .
$$

We will find the invariant $\chi\left(\mathcal{O}_{C}\right)$ convenient since [17, p.261] it is constant on flat families. Thus for curves in a smooth surface $Y, \chi\left(\mathcal{O}_{C}\right)$ depends only on the equivalence class of the divisor of $C$, and is easy to compute. In particular - a fact of which we will make constant use - it takes the same value for any two fibres of a map $Y \rightarrow S$. We also have the adjunction formula

$$
0=2 \chi\left(\mathcal{O}_{C}\right)+C^{2}+C . K_{Y}
$$

where . denotes intersection of divisors and $K_{Y}$ the canonical divisor; and the formula

$$
\chi\left(\mathcal{O}_{A+B}\right)=\chi\left(\mathcal{O}_{A}\right)+\chi\left(\mathcal{O}_{B}\right)-A \cdot B,
$$

which follows from the adjunction formula.

\section{Fibrations with smooth general fibre}

We now suppose $\pi: Y \rightarrow S$ a map with smooth general fibre from a smooth surface $Y$ to a smooth curve $S$, all defined over the algebraically closed field $k$. Over each closed point $s \in S$ we have the fibre $Y_{s}$; over the generic point we also have a fibre, which is a variety defined over $k(S)$; we denote it by $Y_{\text {gen }}$. It 
is usually more geometrical to work over algebraically closed fields; the variety obtained by extending the ground field to the separable algebraic closure is called the geometric generic fibre: we will denote it by $Y_{g g e n}:=Y_{g e n} \otimes_{k(S)} \overline{k(S)}$. Our present hypothesis is that $Y_{s}$ is smooth for all but finitely many $s$, or equivalently that $Y_{\text {ggen }}$ is smooth.

In characteristic zero, the additive property of the Euler characteristic provides a formula

$$
\chi(Y)-\chi\left(Y_{g g e n}\right) \chi(S)=\sum_{s}\left(\chi\left(Y_{s}\right)-\chi\left(Y_{g g e n}\right)\right),
$$

where there are only finitely many non-vanishing terms on the right hand side. For reduced fibres we may write (using $(3)) \chi\left(Y_{s}\right)-\chi\left(Y_{\text {ggen }}\right)=\mu\left(Y_{s}\right)$; for non-reduced fibres various formulae may be inferred by considering the underlying reduced curve $Y_{s, \text { red }}$ and using the facts that $\chi\left(Y_{s}\right)=\chi\left(Y_{s, \text { red }}\right)=2 \chi\left(\mathcal{O}_{Y_{s, \text { red }}}\right)+\mu\left(Y_{s, \text { red }}\right)$. In this section we discuss what becomes of these formulae in the characteristic $p$ case. This involves deep results of Grothendieck and others: it seems worth presenting an account here since many results simplify considerably in this relatively simple situation.

\subsection{Algebra}

Fix a rational point $s \in S$, and write $\mathcal{O}_{S, s}$ for the local ring of $S$ at $s$, with field $k(S)$ of quotients with separable (algebraic) closure $\overline{k(S)}$; write $\widehat{G}$ for the Galois group of $\overline{k(S)}$ over $k(S)$. Choose an extension $\bar{v}_{s}$ to $\overline{k(S)}$ of the discrete valuation $v_{s}$ of $k(S)$ with valuation ring $\mathcal{O}_{S, s}$, and write $G_{s}$, or $G$ for short, for the inertia group of $\bar{v}_{s}$ : then $G_{s}$ is a pro-p-group.

The group $\widehat{G}$ acts on the geometric generic curve $Y_{g g e n}$ and hence on its (étale) cohomology group $H:=H_{e ́ t}^{1}\left(Y_{g g e n} ; \widehat{\mathbb{Z}}_{\ell}\right)$, where $\ell$ is a prime different from $p$. As module over $\widehat{\mathbb{Z}}_{\ell}, H$ is torsion-free with rank $2 g$, where $g$ is the genus of $Y_{\text {ggen }}$. The kernel of the projection $G L_{2 g}\left(\widehat{\mathbb{Z}}_{\ell}\right) \rightarrow G L_{2 g}(\mathbb{Z} / \ell \mathbb{Z})$ is a pro- $\ell$-group, and it follows that the image of $G_{s}$ in the former group maps isomorphically to the second, so in particular is finite. Thus one may work with $H_{e t t}^{1}\left(Y_{g g e n} ; \mathbb{Z} / \ell \mathbb{Z}\right)$ rather than with $H$.

Let $M$ be any finite $G$-module annihilated by $\ell$. Then a subgroup of finite index in $G$ acts trivially on $M$ : write $G_{0}$ for the quotient of $G$ which acts effectively, and $k_{M}$ for the finite extension of $k(S)$ corresponding to $G_{0}$ by Galois duality. Write $v_{M}$ for the restriction to $k_{M}$ of the valuation $\bar{v}_{s}$ of $\overline{k(S)}$ : normalise so that $v_{M}$ has value group $\mathbb{Z}$. Choose a prime element $\varpi$ of $K_{M}$, so $v_{M}(\varpi)=1$. We have the ramification groups

$$
G_{i}:=\left\{\sigma \in G_{0} \mid v_{M}(\sigma(\varpi)-\varpi) \geq i+1\right\} .
$$


The Artin character of $G_{0}$ is now defined by

$$
\alpha(\sigma)=-v_{M}(\sigma(\varpi)-\varpi) \text { if } \sigma \neq 1 ; \sum_{\sigma \in G_{0}} \alpha(\sigma)=0 .
$$

The Swan character $\beta$ is obtained by subtracting from $\alpha$ the character of the augmentation representation. According to Serre (see e.g. [26]), there is a projective $\widehat{\mathbb{Z}}_{\ell}\left[G_{0}\right]$-module $P$ such that $\beta$ is the character of the representation on $P \otimes \widehat{\mathbb{Q}}_{\ell}$. Then 'Serre's measure of the wildness' of $M$ (also called the 'Swan conductor') is

$$
\operatorname{Sw}(M, G):=\operatorname{dim}_{\mathbb{Z} / \ell \mathbb{Z}} \operatorname{Hom}_{\widehat{\mathbb{Z}_{\ell}}\left[G_{0}\right]}(P, M) .
$$

Below we will write $\operatorname{Sw}(M, s)$ for $\operatorname{Sw}\left(M, G_{s}\right)$. It is shown in [22] that, if $M^{i}$ denotes the fixed set of $G_{i}$ acting on $M$, then

$$
\operatorname{Sw}(M, G)=\sum_{i=1}^{\infty} \frac{\left|G_{i}\right|}{\left|G_{0}\right|} \operatorname{dim}_{\mathbb{Z} / \ell \mathbb{Z}}\left(\frac{M}{M^{G_{i}}}\right) .
$$

\subsection{The Ogg-Šafarevič-Grothendieck formula}

The main ingredient of the formulae we are about to describe was first obtained by Ogg [21], and developed in full by Grothendieck [15]; see also Šafarevič [24] and the account [23] of Raynaud.

Let $S$ be (as above) a smooth curve, $F$ a sheaf over $S$ of abelian groups annihilated by $\ell$ : write $F_{\text {ggen }}$ for the stalk over the geometric generic point of $S$. Then the formula is $[15,7.2]$

$$
\chi(S ; F)-\chi(S) \operatorname{dim} F_{g g e n}=\sum_{s \in|S|}\left(\operatorname{dim} F_{s}-\operatorname{dim} F_{g g e n}-\operatorname{Sw}\left(F_{g g e n}, s\right)\right),
$$

where all dimensions are over $\mathbb{Z} / \ell$. Strictly, we should take $F$ as an element of the derived category of such sheaves; the dimensions must then be replaced by Euler characteristics.

The Leray spectral sequence of $\pi: Y \rightarrow S$ for the constant sheaf $\mathbb{Z} / \ell$ gives the calculation

$$
\chi(Y ; \mathbb{Z} / \ell)=\sum_{p, q}(-1)^{p+q} \operatorname{dim} H^{p}\left(S ; R^{q} \pi_{*}(\mathbb{Z} / \ell)\right)=\sum_{q}(-1)^{q} \chi\left(S ; R^{q} \pi_{*}(\mathbb{Z} / \ell)\right) .
$$

Applying (6) yields

$$
\chi\left(S ; R^{q} \pi_{*}(\mathbb{Z} / \ell)\right)-\chi(S) \operatorname{dim}\left(R^{q} \pi_{*}(\mathbb{Z} / \ell)\right)_{\text {ggen }}=\sum_{s \in|S|} A_{s}^{q},
$$

where $A_{s}^{q}$ is given by

$$
A_{s}^{q}:=\operatorname{dim}\left(R^{q} \pi_{*}(\mathbb{Z} / \ell)\right)_{s}-\operatorname{dim}\left(R^{q} \pi_{*}(\mathbb{Z} / \ell)\right)_{\text {ggen }}-\operatorname{Sw}\left(\left(R^{q} \pi_{*}(\mathbb{Z} / \ell)\right)_{\text {ggen }}, s\right)
$$




$$
=\operatorname{dim} H^{q}\left(Y_{s} ; \mathbb{Z} / \ell\right)-\operatorname{dim} H^{q}\left(Y_{\text {ggen }} ; \mathbb{Z} / \ell\right)-\operatorname{Sw}\left(H^{q}\left(Y_{\text {ggen }} ; \mathbb{Z} / \ell\right), s\right) .
$$

Substituting in (7) gives

$$
\chi(Y ; \mathbb{Z} / \ell)=\chi(S) \chi\left(Y_{\text {ggen }} ; \mathbb{Z} / \ell\right)+\sum_{s \in|S|}\left(\chi\left(Y_{s} ; \mathbb{Z} / \ell\right)-\chi\left(Y_{\text {ggen }} ; \mathbb{Z} / \ell\right)-a_{s}\right)
$$

where $a_{s}:=\sum_{q}(-1)^{q} \mathrm{Sw}\left(H^{q}\left(Y_{g g e n} ; \mathbb{Z} / \ell\right), s\right)$. This is essentially the main result of $[12]$.

When the fibres are curves, this simplifies since $[12,1.6]$ if the fibres of $\pi$ are smooth of dimension $n$, then $\operatorname{Sw}\left(H^{q}\left(Y_{\text {ggen }} ; \mathbb{Z} / \ell\right), s\right)$ vanishes unless $0<q<2 n$, so as $n=1$ the only non-vanishing term is for $q=1$. Thus we have

$$
\chi(Y)=\chi(S) \chi\left(Y_{g g e n}\right)+\sum_{s \in|S|}\left(\chi\left(Y_{s}\right)-\chi\left(Y_{g g e n}\right)+\operatorname{Sw}\left(H^{1}\left(Y_{g g e n}, s\right)\right)\right.
$$

where, as from now on, we omit explicit mention of the coefficients, which will remain $\mathbb{Z} / \ell$ throughout.

\subsection{Vanishing cycles}

A general theory of vanishing cycles, due to Grothendieck, is developed by Deligne in [9]. Again we require only a small part of the general theory. Fix a (rational) point $s \in|S|$ (this already simplifies half the notation); the theory is set in the context of the spectrum of the henselisation of $\mathcal{O}_{S, s}$, which we denote by $S_{l o c}$ : it contains the closed point $s$, the generic point $\eta$, and the geometric generic point $\bar{\eta}$; and we have a map $S_{l o c} \rightarrow S$, inducing $Y_{l o c} \rightarrow Y$, say, with fibres $Y_{s}, Y_{\eta}$ and $Y_{\bar{\eta}}$ which we can identify with the $Y_{s}, Y_{\text {gen }}$ and $Y_{\text {ggen }}$ discussed previously.

The first step is to define a functor $\Psi$ from (complexes of) sheaves over $Y_{l o c}$, annihilated by some $\ell^{n}$, to (complexes of) sheaves over $Y_{s} \times S_{l o c}$. To achieve this, one must observe that giving a sheaf $K$ over $Y_{s} \times S_{l o c}$ is equivalent to giving a triple $\left(K_{s}, K_{\eta}, \phi\right)$ with $K_{s}, K_{\eta}$ sheaves over $Y_{s}$ and $\phi$ a morphism from $K_{s}$ to $K_{\eta}$ with image contained in the invariants under the action on the latter of the group $G_{s}$ or Galois group $G_{0}$. There is then a (split) exact sequence $0 \rightarrow(s \circ p)^{*} K_{s} \rightarrow K_{\eta} \rightarrow \Phi(K) \rightarrow 0$, inducing an exact sequence (1.4.2.2 loc. cit.) of cohomology of $Y_{s}$.

Given a sheaf $F$, Deligne defines (1.3, loc. cit.) $\Psi F_{s}:=\left.F\right|_{s}, \Psi F_{\eta}$ is the pullback of the push forward to $Y$ of $\left.F\right|_{\bar{\eta}}$, and $\phi$ is constructed using adjunction morphisms. The above short exact sequence of sheaves must be considered in the derived category as an exact triangle (2.1.2.4 loc. cit.). Further work leads to the identification (2.1.8.9 loc. cit.) of its cohomology exact sequence with

$$
\cdots \rightarrow H^{i}\left(Y_{s} ; F\right) \rightarrow H^{i}\left(Y_{\bar{\eta}} ; F\right) \rightarrow H^{i}\left(Y_{s} ; \Phi\right) \rightarrow H^{i+1}\left(Y_{s} ; F\right) \rightarrow \cdots
$$


where $\Phi$ denotes $\Phi(\Psi F)$. It is also shown that $\Phi$ is supported on the singular set of the map $f$ and (2.4.2 loc. cit.) that if $F$ is a constructible sheaf of $\mathbb{Z} / \ell^{n}$ modules (with $\ell \neq p$ ) which is locally constant at the isolated singular point $x$ of $f$, then the stalk $\Phi_{x}$ of $\Phi$ at $x$ is a finite abelian group.

We are interested in the case when $F$ is a constant sheaf $\mathbb{Z} / \ell^{n}$ and the fibres $Y_{s}$ are curves. The exact sequence shows that $\chi\left(Y_{\bar{\eta}}\right)-\chi\left(Y_{s}\right)=\chi\left(Y_{s} ; \Phi\right)$. Since $\pi_{*}(\mathbb{Z} / \ell) \cong(\mathbb{Z} / \ell), H^{0}\left(Y_{s} ; \Phi\right)$ vanishes. Also $H^{2}\left(Y_{s} ; \Phi\right)$ is trivial: we may argue by duality, or, perhaps more convincingly, since at least one component of $Y_{s}$ must have multiplicity prime to $\ell$. Thus there is only one non-vanishing module of vanishing cycles, and we write $V_{s}$ for $H^{1}\left(Y_{s} ; \Phi\right)$.

Since forming Swan conductors is purely algebraic and additive, and vanishes on the cohomology of $Y_{s}$ where the action of $G$ is trivial, we have

$$
\operatorname{Sw}\left(H^{*}\left(Y_{g g e n}\right), s\right)=\operatorname{Sw}\left(H^{*}\left(Y_{s} ; \Phi\right), s\right)=-\operatorname{Sw}\left(H^{1}\left(Y_{s} ; \Phi\right), s\right)=-\operatorname{Sw}\left(V_{s}, s\right) .
$$

Substituting in (8), we obtain

$$
\chi(Y)=\chi(S) \chi\left(Y_{g g e n}\right)+\sum_{s \in|S|}\left(\operatorname{dim} V_{s}+\operatorname{Sw}\left(V_{s}, s\right)\right) .
$$

Since $\Phi$ is supported on the singular set, if $Y_{s}$ is reduced, so has isolated singularities, $\Phi$ is supported on a finite set, so $V_{s}$ splits as a sum of local contributions $V_{s}=\oplus V_{x}$.

\subsection{The degree of the discriminant}

We turn to global consideration of the map $\pi: Y \rightarrow S$ with $Y, S$ smooth and complete and with the generic fibre also smooth, and apply Example 14.1.5 of Fulton's book [13]. We have a proper morphism $\pi$; the induced map $d \pi: T_{Y} \rightarrow$ $\pi^{*} T_{S}$ determines a section $\sigma$ of $\pi^{*} T_{S} \otimes T_{Y}^{\vee}$. The top Chern class $Z(\sigma)$ is a 0 -cycle class on the singular set of $\pi$, with degree $\operatorname{deg} Z(\sigma)=\chi(Y)-\chi(S) \chi\left(Y_{\text {ggen }}\right)$. The image under $\pi$ of the class of $Z(\sigma)$ is a divisor $D_{\pi}$ on $S$, thus

$$
\operatorname{deg} D_{\pi}=\operatorname{deg} Z(\sigma)=\chi(Y)-\chi(S) \chi\left(Y_{g g e n}\right) .
$$

It was shown by Bloch [2], sharpening a result of Deligne [10] and also giving an interpretation of (8), that the degree of $D_{\pi}$ at a point $s \in|S|$ is

$$
D(s):=\operatorname{deg}_{s}\left(D_{\pi}\right)=\chi\left(Y_{s}\right)-\chi\left(Y_{g g e n}\right)-\operatorname{Sw}\left(H^{*}\left(Y_{g g e n}, s\right)\right) .
$$

Bloch also interpreted this number as a cycle-theoretic self-intersection of the diagonal $\Delta_{Y}$.

We have seen in (9) that in the case of curves, this in turn reduces to

$$
D(s)=\operatorname{dim} V_{s}+\operatorname{Sw}\left(V_{s}, s\right) .
$$


It follows, in particular, that $D(s) \geq 0$, and vanishes if and only if $V_{s}$ is trivial.

We first discuss the case of an isolated critical point. In [10], Deligne defines the numbers of ordinary, wild or total vanishing cycles at an isolated singular point $x$ respectively to be the numbers $\operatorname{dim} V_{x}, \operatorname{dim} \operatorname{Sw}\left(V_{x}, s\right)$ and their sum, and proves (Theorem 2.4 loc. cit.) that the total number of vanishing cycles at $x$ is equal to the Milnor number of $\pi$ at $x$, defined in the usual way by taking local coordinates $(u, v)$ on $Y$ at $x$ and $t$ on $S$ at $s=f(x)$ and setting $f:=t \circ \pi$ and

$$
\mu_{x}(\pi):=\operatorname{dim} \mathcal{O}_{u, v} /\langle\partial f / \partial u, \partial f / \partial v\rangle .
$$

That this is also the local degree of $Z(\sigma)$ is shown by Bloch. Now the ordinary number of vanishing cycles at $x$ is equal to $\operatorname{dim} V_{x}=\mu_{x}\left(Y_{s}\right)$. The relation between the two definitions of Milnor number at an isolated singularity is thus clarified as follows. The Milnor number $\mu_{x}\left(Y_{s}\right)$ of the fibre at $x$ is the dimension of the space $V_{x}$ of vanishing cycles. The inertia group $G_{s}$ of $s=\pi(x)$ acts on this space, and the Milnor number of the map $\pi$ is given by

$$
\mu_{x}(\pi)=\mu_{x}\left(Y_{s}\right)+\operatorname{Sw}\left(V_{x}, s\right) .
$$

If the fibre $Y_{s}$ is reduced, $D(s)$ is the sum of the $\mu_{x}(\pi)$ over singular points $x \in\left|Y_{s}\right|$ : the decomposition into tame and wild cycles is given by (12).

Suppose in addition that $D(s)=0$. Then there are no singular points; if $Y_{\text {ggen }}$ is connected, so is $Y_{s}$, and it has the same genus. Over $\mathbb{C}$, we can then apply Ehresmann's fibration theorem to deduce that $\pi$ is locally topologically trivial at $s$. In finite characteristic, $\pi$ is still locally trivial in some sense which we do not attempt to make precise.

If $Y_{s}$ is not reduced, to obtain a local formula we need a term corresponding to the 1-dimensional part of the singular locus. Globally, Iversen [18] obtained a formula which may be written as

$$
\chi(Y)-\chi\left(Y_{g g e n}\right) \chi(S)=K_{Y} \cdot R-R^{2}+\sum_{x \in|Y|} \mu_{x}^{r e d}(\pi),
$$

where $R$ denotes the ramification divisor, $K_{Y}$ the canonical class, and $\mu_{x}^{r e d}(\pi)$ is defined as follows. As in the case when $x$ is an isolated singular point, we take local coordinates $(u, v)$ on $Y$ at $x$ and $t$ on $S$ at $s=\pi(x)$, and set $f=t \circ \pi$. The partial derivatives $\partial f / \partial u, \partial f / \partial v$ do not generate an ideal of finite codimension in the local ring $\mathcal{O}_{x}$ : their highest common factor $h$ vanishes along the singular locus. We set $a^{u}:=(\partial f / \partial u) / h, a^{v}:=(\partial f / \partial v) / h$, and define

$$
\mu_{x}^{r e d}(\pi):=\operatorname{dim} h \mathcal{O}_{u, v} /\langle\partial f / \partial u, \partial f / \partial v\rangle=\operatorname{dim} \mathcal{O}_{u, v} /\left\langle a^{u}, a^{v}\right\rangle .
$$

The right hand side of (13) is easily expressed as a sum over fibres, and the natural interpretation was achieved by Sun [28] by showing that

$$
D(s)=K_{Y} \cdot R_{s}-R_{s}^{2}+\sum_{x \in\left|Y_{s}\right|} \mu_{x}^{r e d}(\pi)
$$


where $R_{s}$ denotes the part of $R$ lying in the fibre over $s$.

The divisor $R$ is supported on the union of multiple components of the fibres. Let $C_{i}$ be a component of $Y_{s}$, with multiplicity $m_{i}>1$. Let $r_{i}$ be the multiplicity of $C_{i}$ as component of $R$. In local coordinates at a point of $T$ we may write

$$
(t \circ \pi)(u, v)=f(u, v)=u^{m_{i}} \phi(u, v) \text {. }
$$

The definition of $R$ amounts to taking it as defined by the highest common factor of $\partial f / \partial u$ and $\partial f / \partial v$.

Lemma 2.1 If $m_{i}$ is not divisible by $p$ (e.g. if $p=0$ ) then $r_{i}=m_{i}-1$. If $p \mid m_{i}$ then $r_{i}=m_{i}$ unless we can express $\phi$ in the form $\alpha\left(v^{p}\right)+u^{2} \beta(u, v)$, when $r_{i}>m_{i}$.

This follows by an easy calculation. In the first case we have non-zero terms involving $u^{m_{i}-1}$; in the second, if the coefficients of all terms $u^{m_{i}} v^{*}$ in both $\partial f / \partial u$ and $\partial f / \partial v$ vanish, $\phi$ must be as stated.

In the case when $C_{i}$ is a vertical exceptional curve arising in a blowing-up, we take local coordinates and suppose, reverting to the notation of 1.2 and setting $\pi(x)=(f(x): g(x))$, that $f, g$ have respective orders $m+M, m$. Substituting $v=u w$ gives $f / g=\left(u^{M}\left(f_{m+M}(1, w)+O(u)\right) /\left(g_{m}(1, w)+O(u)\right)\right.$, where $O(u)$ denotes terms divisible by $u$. Thus the above invariant $m_{i}$ is here identified with $M$. For the final case in the lemma to arise we require

$$
\frac{f_{m+M}(1, w)}{g_{m}(1, w)}+O(u)=\alpha\left(w^{p}\right)+u^{2} \beta,
$$

and hence that for some $\gamma(w)$ we have $f_{m+M}(1, w) \cong \gamma^{p} g_{m}(1, w)$.

It would be feasible to push these calculations further.

\subsection{The Euler characteristic of the fibre}

We now study a particular (non-reduced) fibre $Y_{s}$. Then we cannot regard the fibration as locally trivial at $s$, and so we expect that $D(s)>0$. By (11), this condition is equivalent to $\chi\left(Y_{\text {ggen }}\right)<\chi\left(Y_{s}\right)=\chi\left(Y_{s, r e d}\right)$. We next investigate whether indeed these inequalities hold.

We begin with some notation. Let $Y_{s}$ have components $C_{i}$, and $Y_{s}=\sum m_{i} C_{i}$ as divisors. Then the underlying reduced curve is $Y_{s, r e d}=\sum C_{i}$. Also write $R_{s}=\sum r_{i} C_{i}$. Thus in the characteristic 0 case, $Y_{s}=R_{s}+Y_{s, r e d}$; in general, $R_{s}+Y_{s, r e d}-Y_{s}$ has non-negative coefficients $r_{i}+1-m_{i}$ which equal 0 only when $m_{i}$ is not divisible by $p$.

Since $Y_{s}$ is numerically equivalent to $Y_{\text {ggen }}$ which is disjoint from $Y_{s}$ we have, for each $j, 0=Y_{s} . C_{j}=\sum m_{i} C_{i} . C_{j}$ and in particular, $Y_{s}^{2}=0$. Below we shall also use without further mention the invariance $\chi\left(\mathcal{O}_{Y_{s}}\right)=\chi\left(\mathcal{O}_{Y_{\text {ggen }}}\right)$, the formula (3) and the adjunction formula (4).

Proposition 2.2 Assume that $Y_{\text {ggen }}$ is connected, and that $Y_{s}$ contains no exceptional curve of the first kind. Exclude the case when $Y_{\text {ggen }}$ and $Y_{s}$ are smooth and rational and $A=0$. Then for any divisor $A$ on $Y_{s}$ with $0 \leq A<Y_{s}$, 
$\chi\left(\mathcal{O}_{A}\right) \geq \chi\left(\mathcal{O}_{Y_{s}}\right)$, with equality only if $A=\lambda Y_{s}$ with $0 \leq \lambda<1$ and $Y_{\text {ggen }}$ is elliptic.

Proof We first briefly recall the (standard) proof that the intersection form restricted to the fibre $Y_{s}$ is negative semi-definite, with radical generated rationally by $Y_{s}$. We have $C_{i} . C_{j} \geq 0$ for $i \neq j$, and $0=C_{i} . Y_{g g e n}=C_{i} \cdot Y_{s}=\sum_{j} m_{j} C_{i} \cdot C_{j}$. Now for any coefficients $x_{i} \in \mathbb{Q}$,

$$
\left(\sum_{i} x_{i} C_{i}\right)^{2}=\sum_{i} x_{i}^{2} C_{i}^{2}+\sum_{i \neq j} x_{i} x_{j} C_{i} . C_{j}=\sum_{i} \frac{x_{i}^{2}}{m_{i}} \sum_{j \neq i}\left(-m_{j} C_{i} . C_{j}\right)+\sum_{i \neq j} x_{i} x_{j} C_{i} . C_{j},
$$

which reduces to

$$
\sum_{i<j}-\frac{\left(m_{j} x_{i}-m_{i} x_{j}\right)^{2}}{2 m_{i} m_{j}} C_{i} \cdot C_{j} \leq 0
$$

For equality to hold, $m_{j} x_{i}-m_{i} x_{j}$ must vanish whenever $C_{i} . C_{j}>0$, i.e. $x_{i} / m_{i}$ takes equal values for components $C_{i}$ and $C_{j}$ that intersect. Since the fibre is connected, (Principle of Connectedness, [17]), all values are equal, so for some $\lambda$ we have $x_{i}=\lambda m_{i}$ for each $i$.

Next let $C$ be a reduced, irreducible component of $Y_{s}$ : we show that $C . K_{Y} \geq 0$. We have $\chi\left(\mathcal{O}_{C}\right)=1$ if $C$ is smooth and rational, $=0$ if $C$ is either nodal or cuspidal rational or smooth elliptic, and otherwise $<0$. If $C$ is a component of $Y_{s}$, then since $C . K_{Y}=-C^{2}-2 \chi\left(\mathcal{O}_{C}\right)$ and $C^{2} \leq 0$, we deduce that if $C . K_{Y}<0$ then either

$\chi\left(\mathcal{O}_{C}\right)=1$ and $C^{2}=0$, so that $C$ is the whole fibre. If $Y_{s}=m C$ we obtain $\chi\left(\mathcal{O}_{Y_{\text {ggen }}}\right)=\chi\left(\mathcal{O}_{Y_{s}}\right)=m$, so that $m$ must be 1 , the fibration rational and $Y_{s}$ a smooth fibre, contradicting our hypothesis; or

$\chi\left(\mathcal{O}_{C}\right)=1$ and $C^{2}=-1$, so that $C$ is smooth and rational and is an exceptional curve of the first kind contained in $Y_{s}$. But by hypothesis this does not exist either.

Moreover, if $C . K_{Y}=0$ then either

$\chi\left(\mathcal{O}_{C}\right)=1$ and $C^{2}=-2$, so $C$ is smooth and rational: $C$ is a '(-2)-curve', or

$\chi\left(\mathcal{O}_{C}\right)=0$ and $C^{2}=0$, hence $Y_{s}$ is a multiple of $C$, and the entire fibre reduces to the curve $C$.

Now let $A=\sum a_{i} C_{i}$ with $0 \leq a_{i} \leq m_{i}$. By the adjunction formula,

$$
2\left(\chi\left(\mathcal{O}_{A}\right)-\chi\left(\mathcal{O}_{Y_{s}}\right)\right)=Y_{s} . Y_{s}+Y_{s} . K_{Y}-A . A-A . K_{Y}
$$

But the intersection form restricted to a fibre is negative semi-definite, so $A$. $A \leq$ 0 , while $Y_{s} \cdot Y_{s}=0$. We thus obtain

$$
2\left(\chi\left(\mathcal{O}_{A}\right)-\chi\left(\mathcal{O}_{Y_{s}}\right)\right)=(-A \cdot A)+\sum_{i}\left(m_{i}-a_{i}\right) C_{i} \cdot K_{Y}
$$

Here each term on the right is non-negative. Thus for equality to hold, each term must vanish. Since $A^{2}=0, A$ is a (rational) multiple of $Y_{s}$, so for some 
$\lambda, a_{i}=\lambda m_{i}$ for each $i$. It follows from our hypothesis that $\lambda<1$, so $m_{i}>a_{i}$ for each $i$. Hence each $C_{i} \cdot K_{Y}$ must vanish, and we have $Y_{s} \cdot K_{Y}=0$, thus the fibration is elliptic.

We can be more precise about the case of equality.

Corollary 2.3 If equality holds, $Y_{s}$ has Kodaira type $m I_{b}$ for some $m \geq 2, b \geq 0$. Thus $Y_{s}=m \Theta_{b}$ for some b, where

$\Theta_{0}$ is smooth elliptic,

$\Theta_{1}$ is nodal rational,

$\Theta_{2}$ consists of two smooth rational curves meeting transversely in 2 points, for $b \geq 3, \Theta_{b}$ is a cycle of $b$ (-2)-curves, each component meeting the next.

This follows from the enumeration of types of (non-reduced) fibre in elliptic fibrations by Kodaira [19] in characteristic 0 and Bombieri and Mumford [3] in general.

We next deal with exceptional curves contained in the fibre.

Proposition 2.4 Assume that $Y_{\text {ggen }}$ is connected. Suppose $Z$ obtained by successively collapsing exceptional curves of the first kind in the fibre over s. Then $\left(\chi\left(\mathcal{O}_{Y_{s, \text { red }}}\right)-\chi\left(\mathcal{O}_{Y_{s}}\right)\right) \geq\left(\chi\left(\mathcal{O}_{Z_{s, \text { red }}}\right)-\chi\left(\mathcal{O}_{Z_{s}}\right)\right)$, and hence is non-negative.

Proof Suppose there is an exceptional curve of the first kind $E \subset Y_{s}$. Write $Y_{s, \text { red }}=B+E$, and let $M:=E . B$. Collapsing $E$ to a point gives a surface $Y^{\prime}$, a factorisation $Y \rightarrow Y^{\prime} \rightarrow S$ of $\pi$, and hence a fibre $Y_{s}^{\prime}$ with $Y_{s, r e d}^{\prime}=B^{\prime}$, the image of $B$, so that $B^{\prime}$ has multiplicity $M$ at the image $e$ of $E$ and hence strict transform $B+M E$. The standard rules for blowings up give

$B . K_{Y}=B^{\prime} . K_{Y^{\prime}}+M, \quad B^{2}=B^{\prime 2}-M^{2}, \quad$ so $\quad \chi\left(\mathcal{O}_{B}\right)=\chi\left(\mathcal{O}_{B^{\prime}}\right)+\left(M^{2}-M\right) / 2$.

Hence

$$
\chi\left(\mathcal{O}_{Y_{s, r e d}}\right)=\chi\left(\mathcal{O}_{B}\right)+\chi\left(\mathcal{O}_{E}\right)-B \cdot E=\chi\left(\mathcal{O}_{B^{\prime}}\right)+\frac{1}{2}\left(M^{2}-M\right)+1-M,
$$

which is equal to $\chi\left(\mathcal{O}_{Y_{s, r e d}^{\prime}}\right)+\frac{1}{2}(M-1)(M-2) \geq \chi\left(\mathcal{O}_{Y_{s, r e d}^{\prime}}\right)$. If $Y_{s}^{\prime}$ also contains an exceptional curve, we may repeat the process, which must terminate at some surface $Z$, say, with fibre $Z_{s}$. It follows by induction that $\chi\left(\mathcal{O}_{Y_{s, \text { red }}}\right) \geq \chi\left(\mathcal{O}_{Z_{s, \text { red }}}\right)$, while $\chi\left(\mathcal{O}_{Z_{s}}\right)=\chi\left(\mathcal{O}_{Z_{\text {ggen }}}\right)=\chi\left(\mathcal{O}_{Y_{\text {ggen }}}\right)=\chi\left(\mathcal{O}_{Y_{s}}\right)$, since the generic fibre is unaltered by the collapsing. The final assertion follows by Proposition 2.2.

The conditions for equality here may be inferred from the previous result and the fact that we then need $M=1$ or $M=2$ at each step of the blowing down process.

Proposition 2.5 Assume that $Y_{\text {ggen }}$ is connected, and that $Y_{s}$ is non-reduced. Then $\chi\left(Y_{s}\right)>\chi\left(Y_{\text {ggen }}\right)$ unless each of $Y_{s}$ and $Y_{\text {ggen }}$ is a smooth elliptic curve. 
Proof By Proposition 2.4, we have $\chi\left(\mathcal{O}_{Y_{s, \text { red }}}\right) \geq \chi\left(\mathcal{O}_{Y_{s}}\right)$, and this in turn is equal to $\chi\left(\mathcal{O}_{Y_{\text {ggen }}}\right)$. Hence

$\chi\left(Y_{s, r e d}\right)=2 \chi\left(\mathcal{O}_{Y_{s, r e d}}\right)+\mu\left(Y_{s, r e d}\right) \geq 2 \chi\left(\mathcal{O}_{Y_{\text {ggen }}}\right)+\mu\left(Y_{s, r e d}\right)=\chi\left(Y_{\text {ggen }}\right)+\mu\left(Y_{s, r e d}\right)$.

We thus obtain $\chi\left(Y_{s}\right)=\chi\left(Y_{s, \text { red }}\right)>\chi\left(Y_{\text {ggen }}\right)$ unless first, we have equality above, so $Y_{\text {ggen }}$ is elliptic, and secondly, $\mu\left(Y_{s, r e d}\right)=0$, so $Y_{s, r e d}$ is smooth. The result follows.

According to [25], this result is well known. We include the proof for completeness.

We conclude this section with a note about wild ramification in the case of non-isolated singularities. In view of Lemma 2.1 we can regard the ramification at $C_{i}$ as wild when $p \mid m_{i}$, though the relation of this wildness condition to calculation of the Swan conductor is not clear.

Lemma 2.6 Suppose $R_{s}$ is tame, i.e. that no $m_{i}$ is divisible by $p$. Then

$$
\mathrm{Sw}\left(V_{s}, s\right)=\sum_{x \in\left|Y_{s}\right|}\left(\mu_{x}^{r e d}(\pi)-\mu_{x}\left(Y_{s, r e d}\right)\right) .
$$

Proof It follows from our hypothesis that $R_{s}=Y_{s}-Y_{s, r e d}$. Hence the expression for $D(s)$ given by (14) is equal to

$$
K_{Y} \cdot\left(Y_{s}-Y_{s, r e d}\right)-\left(Y_{s}-Y_{s, r e d}\right)^{2}+\sum_{x \in\left|Y_{s}\right|} \mu_{x}^{r e d}(\pi) \text {. }
$$

Using (3) and (4), the expression for $D(s)$ given by (10) may be expressed as

$$
\mu\left(Y_{s, \text { red }}\right)-K_{Y} \cdot Y_{s, \text { red }}-Y_{s, r e d}^{2}+K_{Y} \cdot Y_{\text {ggen }}+Y_{\text {ggen }}^{2}+\operatorname{Sw}\left(V_{s}, s\right) .
$$

Comparing these two, and using $K_{Y} \cdot Y_{s}=K_{Y} \cdot Y_{\text {ggen }}$ and $Y_{s}^{2}=0=Y_{s} \cdot Y_{s, \text { red }}=$ $Y_{g g e n}^{2}$, we see that the equation simplifies to the form stated.

If $R_{s}$ is not tame, and some component through $x$ has multiplicity divisible by $p$, then the local equation has the form $f=g_{1}^{p} g_{2}$, and differentiating gives $\partial f=$ $g_{1}^{p} \partial g_{2}$, so we expect no close relation between $\mu_{x}^{r e d}(\pi)$ and $\mu_{x}\left(Y_{s, r e d}\right)$.

\section{Pencils of curves and Bertini's theorem}

Consider a smooth surface $X_{0}$ with a pencil of curves $\left\{\Gamma_{t}\right\}$ whose generic member $\Gamma_{g e n}$ is reduced and irreducible, but may be singular. If the field $k$ has finite characteristic $p$, Bertini's theorem in its original form is not always valid. For example, for the plane pencil $t_{0}\left(x_{0}^{3}+x_{1}^{2} x_{2}\right)+t_{1} x_{2}^{3}$ in characteristic 2 , the curve $\Gamma_{t}$ has a singular point at $\left(0: u_{0}: u_{1}\right)$ where $\left(u_{0}^{2}: u_{1}^{2}\right)=\left(t_{1}: t_{0}\right)$. A detailed study of systems of singular cubics appears in [3].

The correct formulation of Bertini's theorem for this case was found by Zariski in 1944 [30]: the introduction by Mumford to the first part of Zariski's collected works gives a useful account of Zariski's achievement in modern terminology. 
The key is to consider the generic curve of the pencil as defined over the field $k(t)$. Since this field is not perfect, many of the familiar results of algebraic geometry do not hold without slight modifications. In particular, two of the traditional definitions of a 'simple point' on an algebraic variety are not here equivalent: if the variety is defined by equations whose differentials at that point cut out a tangent space of the same dimension as the variety it is called smooth there; if the local ring of the variety at that point is regular, it is called a regular point.

Then Zariski's version of Bertini's theorem states that the generic curve $\Gamma_{g e n}$ of the pencil is regular outside the set of base points of the pencil. It is important here to distinguish between $\Gamma_{g e n}$ and $\Gamma_{g g e n}$. Following the procedure in $\S 1.2$, we study $\Gamma_{g e n}$ by blowing up all the base points of the pencil to obtain a pencil of curves $Y_{s}$ on a surface $Y$ having no base points. It follows from the theorem that $Y_{\text {gen }}$ is regular (at all points).

It follows from elementary results about local rings that for curves the conditions 'regular' and 'normal' are equivalent. A function field of transcendence degree 1 over any field has an essentially unique normal model. The arguments leading to the proof of the Riemann-Roch theorem go through over any field (see e.g. Artin [1]). It follows from this theorem that for an irreducible normal curve $\mathrm{C}$ the genus and arithmetic genus agree, $g(C)=p_{a}(C)$.

The genus of a curve over a non-perfect field is not invariant under inseparable change of ground field (the first paper on this topic seems to be Tate [29], where it is shown that $g(C)-g(\bar{C})$ is a multiple of $\frac{p-1}{2}$ ). (Since separable base change commutes with integral closure, and so preserves normality, this cannot affect the genus.) We may control the genus as follows. Since $\chi\left(\mathcal{O}_{C}\right)$ is constant in flat families (the constancy including both closed and non-closed points), it is invariant under change of ground field. On ground field extension however we may acquire non-regular points, and have to blow these up. If $C_{1}$ is the result of blowing up a closed point $P$ on $C$, then taking Euler characteristics of the exact sequence

$$
0 \rightarrow \mathcal{O}_{C} \rightarrow f_{*} \mathcal{O}_{C_{1}} \rightarrow\left(\sum_{f(Q)=P} \mathcal{O}_{1}(Q)\right) / \mathcal{O}_{C}(P) \rightarrow 0
$$

we see that $\chi\left(\mathcal{O}_{C_{1}}\right)-\chi\left(\mathcal{O}_{C}\right)$ is the length of $\left(\sum_{f(Q)=P} \mathcal{O}_{1}(Q)\right) / \mathcal{O}_{C}(P)$; and this length is equal to $\frac{1}{2} m_{P}\left(m_{P}-1\right)[K(P): K]$ where $m_{P}$ is the multiplicity of $C$ at the point $P$ and $K$ the field of definition of $C$ (see Deligne [8] for a full treatment of this situation). The effect on the genus is thus to subtract the sum of the terms $\frac{1}{2} m_{P}\left(m_{P}-1\right)[K(P): K]$ corresponding to the points that have to be blown up. If we are extending the ground field to be algebraically closed, then all singular infinitely near points need to be resolved, and by (1) the sum is the double point number $\delta(\bar{C})$ : thus for $C$ regular, $g(\bar{C})=g(C)-\delta(\bar{C})$. Alternatively we may argue that $g(\bar{C})=p_{a}(\bar{C})-\delta(\bar{C})$, while $p_{a}(\bar{C})=p_{a}(C)=g(C)$.

Thus blowing up the base points of the pencil gives a regular generic fibre $Y_{\text {gen }}$, however $Y_{\text {ggen }}$ may have singular points; necessarily only defined over inseparable 
extensions of $k(t)$. We have $g\left(Y_{\text {ggen }}\right)=g\left(Y_{\text {gen }}\right)-\delta\left(Y_{g g e n}\right)$. Artin uses the word conservative for the case when the genus is stable by base change: we see that this is equivalent to $Y_{\text {ggen }}$ being non-singular. We may use the word radical for the opposite case.

If $\Gamma_{t}$ has a singular point which moves with $t$, the pencil is radical. If $f_{0}=$ $a b_{0}^{p}+c_{0} h^{2}$ and $f_{1}=a b_{1}^{p}+c_{1} h^{2}$ as polynomials on the plane $P^{2}$, then the general member $t_{0} f_{0}+t_{1} f_{1}$ of the pencil is singular at points where $0=t_{0} b_{0}^{p}+t_{1} b_{1}^{p}=h$. Thus any curve $h=0$ may arise as a locus of singular points. It may be true that all cases may be put in this form. We can prove this if $h$ is linear and have a sketch of proof whenever $h=0$ is an irreducible rational curve.

Variable singular points may also arise on blowing up: consider an isolated base point of multiplicity $m$, with local coordinates $(u, v)$ as usual. The first blowup produces an exceptional curve with coordinate $(\xi: \eta)$ : assume it dicritical. The base points on this curve correspond to common zeros of $f_{m}$ and $g_{m}$. The point $(1: 0)$ is singular on the blow-up of $f=0$ if $v^{2}$ divides $f_{m}$ and $v$ divides $f_{m+1}$. We have already seen that the exceptional curve is inseparable if $f_{m}$ and $g_{m}$ are both $p^{\text {th }}$ powers, or more generally if $f_{m} / g_{m}$ is so; if in addition $g_{m} f_{m+1}=f_{m} g_{m+1}$ (e.g. if $f_{m+1}=g_{m+1}=0$ ), then each point of the exceptional curve is singular on some member of the pencil, which is thus radical.

The radical pencils were termed 'supercuspidal' and studied in [27], where some results of [3] were generalised. Observe that (since $k$ is perfect) all elements of $k[t]$ become $p^{t h}$ powers in $k\left[t^{1 / p}\right]$, so all elements of $k(t)$ become $p^{t h}$ powers in $k\left(t^{1 / p}\right)$. Thus if $x$ is algebraic over $k(t)$ with minimal equation $f\left(x^{p^{r}}\right)=0$ where $f$ is separable, there is a separable $g$ over $k\left(t^{p^{-r}}\right)$ with $f\left(x^{p^{r}}\right) \cong g(x)^{p^{r}}$, so $x$ is separable over $k\left(t^{p^{-r}}\right)$. Thus any finite extension of $k(t)$ may be regarded as a separable extension of $k\left(t^{p^{-r}}\right)$ for some $r$.

Consider the sequence $S_{r} \rightarrow S_{r-1} \cdots \rightarrow S_{1} \rightarrow S_{0}=S$ with each $S_{i}$ isomorphic to $P^{1}$ and each map given by the Frobenius map $F(t)=t^{p}$. We can pull back the map $\pi_{0}: Y_{0} \rightarrow S_{0}$ to give maps $\pi_{k}: Y_{k} \rightarrow S_{k}$. These are not given by pencils, but are certainly families of curves. If the field generated by all coordinates of all points blown up in the minimal resolution of $Y_{\text {ggen }}$ is $K$, then by the above, $K$ is separable over $k\left(t^{p^{-r}}\right)$ for some $r$, so the normalisation of the generic fibre of $Y_{r}$ is smooth.

Shimada's main result gives a normal form for the local behaviour at a generic point on the singular curve in the case $r=1$.

Theorem 3.1 [27] In suitable local coordinates in $Y$, if the above parameter $r$ is $1, \pi$ is given at a generic point on a singular curve by either

(a) if $Y_{1}$ is separable over $S_{1}, \pi(x, y)=x^{p}-y^{m}$ for some $m>1$ prime to $p$;

(b) if the inseparable degree is $q, \pi(x, y)=x^{p}+y^{q}+x^{p m} y$ for some $m>0$.

We have not succeeded in obtaining a good insight into the structure of radical pencils. Perhaps the most natural construction is to resolve the singularities of 
the generic fibre. There are finitely many of these: one may make a base change, extending $k(t)$ to a field over which they (and all infinitely near points along which blowing up is required) are well defined, so that the field extension defines a finite map $S_{1} \rightarrow S_{0}$. Note also that geometrically, blowing up a point whose coordinates depend on $t$, is perhaps best understood as taking the strict transform of $X_{1} \subset X_{0} \times S_{0}$ under the blow-up of a section of $X_{0} \times S_{0} \rightarrow S_{0}$. We can combine this with a base change by blowing up $X_{0} \times S_{0}$ along the curve consisting of pairs $(Q, t)$ such that $\Gamma_{t}$ is singular at $Q$.

Eventually one must arrive at a smooth surface $Y_{1}$, a map $\pi_{1}: Y_{1} \rightarrow S_{1}$, and a collection of curves $E_{r}$ in $Y_{1}$ consisting of all the exceptional curves arising during the above procedure. We can call a point of $S_{1}$ or $S_{0}$ atypical if it is a critical value of the projection of any of the $E_{r}$, or the image of an intersection point of two distinct curves $E_{r}$. In some sense the other values should all be typical. But we see no way to control this sequence of operations: at the least, one would need a rather good understanding of equisingularity for the singularities deforming those of the generic fibre. We thus restrict from now on to the conservative case.

\section{Conservative pencils}

We saw in $\S 3$ that conservative pencils are characterised by the property that if all the base points of the pencil are blown up, the generic fibre becomes smooth: this property is automatic in characteristic 0 . We shall assume from now on that this holds and that, moreover, the generic fibre is irreducible: we may then use the results of $\S 2$.

The point of main interest to us is to define what it means for a member of the pencil to be atypical, and to detect such members. For work over $\mathbb{C}$ we take 'atypical' to mean a member at which topological triviality of the family fails. We seek a model which we can follow for the finite characteristic case.

The following was proved in the complex case by Lê and Weber [20] by topological arguments (although that paper was addressed to the local case, the results are applicable globally).

Theorem 4.1 (a) (4.3, loc. cit.) The function $\mu\left(\Gamma_{t}\right)$ (interpreted as $\infty$ if $\Gamma_{t}$ is non-reduced) is an upper semi-continuous function of $t$; and topological triviality holds along the set where it takes its minimum value.

(b) The complement of this set is finite, and consists of values where either $\Gamma_{t}$ is non-reduced or $\chi\left(\Gamma_{t}\right)>\chi\left(\Gamma_{\text {ggen }}\right)$ : such values are called atypical.

(c) $(4.1$, loc.cit.) The value $t$ is atypical if and only if either

$\left(C_{1}\right) Y_{t}$ is singular,

$\left(C_{2}\right) t$ is a branch value for some dicritical exceptional curve, or

$\left(C_{3}\right) Y_{t}$ contains a point of intersection of two dicritical curves.

(d) The pencil is topologically trivial over the complement of the atypical set. 
We proceed to a direct combinatorial proof, valid in all characteristics, of a corresponding result.

Theorem 4.2 Let $\left\{\Gamma_{t}\right\}$ be a pencil of curves in a smooth surface $X$ such that the generic curve $\Gamma_{\text {ggen }}$ is irreducible. Then $\chi\left(\Gamma_{t}\right) \geq \chi\left(\Gamma_{\text {ggen }}\right)$. Equality holds if and only if $Y_{t, r e d}$ is smooth, $C_{2}$ and $C_{3}$ both fail, and either $Y_{t}$ is reduced or $Y_{t, r e d}$ (hence also $Y_{\text {ggen }}$ ) is smooth elliptic.

We begin the proof by finding a formula expressing the difference $\chi\left(\Gamma_{t}\right)-\chi\left(\Gamma_{g g e n}\right)$ as a sum of terms which we will then prove to be non-negative. A consideration of the cases when they vanish will lead to the conclusion. As in $\S 1.2$ we blow up all base points of the pencil to give a map $\pi: Y \rightarrow S$.

We need to take care with notation. Write $Y_{t}$ for the special fibre. As a divisor, we can write this as a sum $\tilde{\Gamma}_{t}+E_{t}$, where $E_{t}$ is the sum of terms corresponding to exceptional curves (for the map $Y \rightarrow X$ ) lying in the fibre $Y_{t}$. We add a further suffix red to refer to the underlying reduced curves. We write $D$ for the union of all dicritical curves $D_{i}$ in $Y$, and define the $\mathbb{Q}$-divisor $D_{Q}$ to be the sum over $i$ of terms $p^{-a_{i}}\left[D_{i}\right]$, where $p^{a_{i}}$ is the degree of inseparability of the projection $D_{i} \rightarrow S$.

Lemma 4.3 We have $\chi\left(\Gamma_{t}\right)-\chi\left(\Gamma_{\text {ggen }}\right)=\sum_{1}^{6} N_{r}$, where

$$
\begin{array}{ll}
N_{1}=2\left(\chi\left(\mathcal{O}_{Y_{t, r e d}}\right)-\chi\left(\mathcal{O}_{Y_{t}}\right)\right), & N_{2}=\mu\left(\tilde{\Gamma}_{t, r e d}\right), \\
N_{3}=\tilde{\Gamma}_{t, r e d} \cdot E_{t, r e d}-\#\left(\tilde{\Gamma}_{t} \cap E_{t}\right), & N_{4}=\tilde{\Gamma}_{t, r e d} \cdot E_{t, \text { red }}-\chi\left(\mathcal{O}_{E_{t, \text { red }}}\right), \\
N_{5}=\#\left(D \cap E_{t}\right)-\chi\left(\mathcal{O}_{E_{t, \text { red }}}\right), & N_{6}=Y_{t} \cdot D_{Q}-\#\left(Y_{t} \cap D\right) .
\end{array}
$$

Proof By $(5), \chi\left(\mathcal{O}_{Y_{t, r e d}}\right)=\chi\left(\mathcal{O}_{\tilde{\Gamma}_{t, \text { red }}}\right)+\chi\left(\mathcal{O}_{E_{t, \text { red }}}\right)-\tilde{\Gamma}_{t, \text { red }} . E_{t, \text { red }}$. Since also $\chi\left(\mathcal{O}_{Y_{\text {ggen }}}\right)=\chi\left(\mathcal{O}_{Y_{t}}\right)$,

$$
\chi\left(\mathcal{O}_{\tilde{\Gamma}_{t, r e d}}\right)-\chi\left(\mathcal{O}_{Y_{\text {ggen }}}\right)=\left(\chi\left(\mathcal{O}_{Y_{t, \text { red }}}\right)-\chi\left(\mathcal{O}_{Y_{t}}\right)\right)-\chi\left(\mathcal{O}_{E_{t, \text { red }}}\right)+\tilde{\Gamma}_{t, \text { red }} \cdot E_{t, \text { red }} .
$$

Now $\chi\left(\tilde{\Gamma}_{t, r e d}\right)=2 \chi\left(\mathcal{O}_{\tilde{\Gamma}_{t, r e d}}\right)+\mu\left(\tilde{\Gamma}_{t, r e d}\right)$, while the generic fibre is smooth, so

$$
\chi\left(\tilde{\Gamma}_{t, r e d}\right)-\chi\left(Y_{\text {ggen }}\right)=2\left(\chi\left(\mathcal{O}_{\tilde{\Gamma}_{t, \text { red }}}\right)-\chi\left(\mathcal{O}_{Y_{\text {ggen }}}\right)\right)+\mu\left(\tilde{\Gamma}_{t, r e d}\right) .
$$

On the other hand, the difference $\chi\left(\tilde{\Gamma}_{t}\right)-\chi\left(\Gamma_{t}\right)$ can be evaluated by counting the numbers of points in preimages under $\tilde{\Gamma}_{t} \rightarrow \Gamma_{t}$. This map is bijective outside the set $B$ of base points of the pencil. We must thus count the number of points of $\tilde{\Gamma}_{t}$ lying over the points of $B$. These are the points of $\tilde{\Gamma}_{t}$ which also lie on one or more exceptional curves. These curves may be dicritical or contained in the fibre $Y_{t}$, and hence in $E_{t}$. Thus we have $\chi\left(\tilde{\Gamma}_{t}\right)-\chi\left(\Gamma_{t}\right)=\#\left(\tilde{\Gamma}_{t} \cap\left(D \cup E_{t}\right)\right)-\# B$. Here we expand \#( $\left.\tilde{\Gamma}_{t} \cap\left(D \cup E_{t}\right)\right)=\#\left(\tilde{\Gamma}_{t} \cap D\right)+\#\left(\tilde{\Gamma}_{t} \cap E_{t}\right)-\#\left(\tilde{\Gamma}_{t} \cap D \cap E_{t}\right)$. We proceed similarly with the generic fibre, save that $E_{g e n}$ is empty. Comparing these shows that $\chi\left(\Gamma_{t}\right)-\chi\left(\Gamma_{g g e n}\right)$ is equal to

$$
\left(\chi\left(\tilde{\Gamma}_{t}\right)-\chi\left(Y_{\text {ggen }}\right)\right)+\left(\#\left(Y_{\text {ggen }} \cap D\right)-\#\left(\tilde{\Gamma}_{t} \cap D\right)\right)-\#\left(\tilde{\Gamma}_{t} \cap E_{t}\right)+\#\left(\tilde{\Gamma}_{t} \cap D \cap E_{t}\right) .
$$


The definition of $D_{Q}$ was so framed that for each dicritical curve $D_{i}$, its intersection number with the fibre at a generic point is $p^{a_{i}}$ so the intersection number $D_{Q} \cdot Y_{\text {ggen }}$ is 1 . Hence $D_{Q} \cdot Y_{\text {ggen }}=\#\left(D \cap Y_{\text {ggen }}\right)$. Since $Y_{t}$ is linearly equivalent to $Y_{g g e n}$, we have $Y_{g g e n} . D=Y_{t} . D$ and so

$$
\#\left(Y_{g g e n} \cap D\right)=Y_{g g e n} . D_{Q}=Y_{t} \cdot D_{Q} .
$$

Substituting the right hand side of (15) for $\chi\left(\mathcal{O}_{\tilde{\Gamma}_{t, \text { red }}}\right)-\chi\left(\mathcal{O}_{Y_{\text {ggen }}}\right)$ in $(16)$, and substituting the result and (18) in (17) gives an expression for $\chi\left(\Gamma_{t}\right)-\chi\left(\Gamma_{\text {ggen }}\right)$ which is the sum of $N_{1}, N_{2}, N_{3}, N_{4}$ and

$$
-\chi\left(\mathcal{O}_{E_{t, r e d}}\right)-\#\left(\tilde{\Gamma}_{t} \cap D\right)+\#\left(\tilde{\Gamma}_{t} \cap D \cap E_{t}\right)+Y_{t} \cdot D_{Q}
$$

But since $\#\left(D \cap Y_{t}\right)=\#\left(D \cap \tilde{\Gamma}_{t}\right)+\#\left(D \cap E_{t}\right)-\#\left(D \cap \tilde{\Gamma}_{t} \cap E_{t}\right)$, this reduces to $N_{5}+N_{6}$.

We next show that each of the terms $N_{1}-N_{6}$ is non-negative. Indeed, the assertion for $N_{1}$ was proved in Proposition 2.4. Non-negativity of $N_{2}$ and $N_{3}$ is immediate, and for $N_{6}$ follows since at each intersection point of $Y_{t}$ with any $D_{i}$, the intersection number is the local degree of the projection of $D_{i}$ on $S$, which is a multiple of $p^{a_{i}}$.

Since the exceptional curves are constructed by a sequence of blowings-up of points in a smooth surface, they (or rather, the dual graph) form a forest with normal crossings. In particular, the curves $E_{t}$ contained in $Y_{t}$ form a forest, which is a union of trees $T$. Each tree $T_{\text {red }}$ consists of smooth rational curves with normal crossings, thus each having $\chi(\mathcal{O})=1$ and each edge of the dual graph corresponds to an intersection number 1 . Since the number of vertices exceeds that of edges by 1 , it follows from (5) that $\chi\left(\mathcal{O}_{T_{\text {red }}}\right)=1$.

As $Y_{t}$ is connected, and $T$ is not the whole fibre, it must meet the union of the others, hence $T \cap \tilde{\Gamma}_{t}$ is non-empty. Thus $T_{\text {red. }} . \tilde{\Gamma}_{t, r e d}-\chi\left(\mathcal{O}_{T_{\text {red }}}\right) \geq 0$, and nonnegativity of $N_{4}$ follows by summing over trees $T$. We also see that non-negativity of $N_{5}$ will follow if each tree $T$ intersects $D$ : we address this next.

We recall from $\S 1.1$ the notion ' $a_{\beta}$ lies above $a_{\alpha}$ ', which gives a partial order on the set of infinitely near points; we say that a set $J$ of infinitely near points is convex if $a_{\alpha}<a_{\beta}<a_{\gamma}$ and $a_{\alpha} \in J, a_{\gamma} \in J$ imply $a_{\beta} \in J$.

Lemma 4.4 For each $T$, every maximal component $E$ of $T$ meets $D$. Hence $T \cap D \neq \emptyset$.

Proof Let $E_{m}$ be a component of $T$ which is maximal in the above partial order. Since $e_{m}$ is a base point, in the surface $X_{m}$ where $E_{m}$ has just been created $E_{m}$ meets $Y_{g g e n}$, in $P$, say. This cannot lie on any other component of $T$ since otherwise blowing it up would disconnect $T$ which, by hypothesis, remains connected in $Y$.

Consider the sequence of blowings-up at points of $E_{m}$ over $P$ in higher surfaces in the sequence until the corresponding point ceases to lie on $Y_{g g e n}$. This produces 
a chain of exceptional curves $A_{1}, A_{2}, \ldots, A_{k}$ with each intersecting $E_{m}$ at $P$ at its first appearance, so that no further blowing up takes place over $P$. Since $T$ is a connected component, $A_{k}$ cannot lie in the fibre $Y_{t}$ : nor can it lie in another fibre, since this is disjoint from $Y_{t}$. Hence $A_{k}$ is dicritical, and the result follows.

This completes the proof of the inequality in Theorem 4.2. We next seek to characterise the cases of equality.

Lemma 4.5 Suppose equality holds in Theorem 4.2. Then

(i) Each component of $Y_{t, \text { red }}$ is smooth, and $Y_{t, r e d}$ has normal crossings. Each component $T$ of $E_{t}$ meets $\tilde{\Gamma}_{t}$ in just one point. $\tilde{\Gamma}_{t}$ has just one component, and the dual graph of $Y_{t}$ is a tree.

(ii) If $Y_{t}$ is not reduced, it has just one component which is smooth, elliptic, and of multiplicity a power of $p$.

(iii) Neither $C_{2}$ nor $C_{3}$ holds. Each tree $T$ meets $D$ in just one point.

(iv) For each $T$, the set of components of $T$ is totally ordered and convex.

Proof (i) As $N_{2}=0, \tilde{\Gamma}_{t, r e d}$ is smooth (and components of $E_{t, r e d}$ are smooth anyway); as $N_{4}=0$, it follows from the above discussion that for each component $T$ of $E_{t}$ we have $T_{r e d} . \tilde{\Gamma}_{t, r e d}=1$, so $T$ meets $\tilde{\Gamma}_{t}$ in just one point, transversely. Thus $Y_{t, \text { red }}$ has normal crossings. Since $Y_{t}$ is connected, it follows that $\tilde{\Gamma}_{t}$ is connected, so has just one component, and the dual graph of $Y_{t}$ is a tree.

(ii) Suppose $Y_{t}$ non-reduced. Since $N_{1}=0$, it follows from Proposition 2.4 that if all exceptional curves of the first kind in $Y_{t}$ are collapsed in turn we obtain a fibre of Kodaira type $m I_{b}$ for some $m \geq 2, b \geq 0$. If $b \geq 1$, the dual graph is not a tree, but contains a cycle (of length $b$ ). Blowing up points to produce exceptional curves will still leave a cycle (perhaps of greater length). Hence this case does not occur.

Since $b=0$, there is a component of $Z_{t}$, hence of $Y_{t}$, of genus 1: this component must be $\tilde{\Gamma}_{t}$. Since by Lemma 1.2 no other component may be exceptional of the first kind, $Y_{t}$ just has one component $C$. Finally, if $C$ has multiplicity $m$ as component of the fibre, and $D$ is dicritical of inseparable degree $p^{i}$, the local intersection number at any point $P \in C \cap D$ satisfies $p^{i}=D . Y_{t}=m D$.C, so $m$ is a power of $p$.

(iii) Next we show that $N_{6}>0$ if and only if either $C_{2}$ or $C_{3}$ holds. Consider a point $P$ of intersection of $Y_{t}$ with a dicritical $D_{i}$. The intersection $D_{i} . Y_{t}$ is $p^{a_{i}}$ if we have no ramification at $t$, otherwise is greater. Thus if $C_{2}$ holds, the point $P$ contributes $>1$ to $Y_{t} \cdot D_{Q}$, and this is also true if $C_{3}$ holds and we have two dicritical curves through $P$. The converse follows by the same argument.

The vanishing of $N_{5}$ shows that each $T$ meets $D$ in just one point.

(iv) Since the partial order on the set of exceptional curves gives this the structure of a 1-way tree, if $T$ is not a chain it has more than one maximal 
element. But the proof of Lemma 4.4 shows that each maximal element meets $D$, so then $\#(T \cap D) \geq 2$, contradicting the above.

Let $\bar{T}$ denote the convex cover of $T$ - i.e. the set of all exceptional curves $C$ such that for some $A, B \in T$ we have $A<C<B$. Since each blow-up produces a component intersecting the union of the preceding ones, the union of all curves in $\bar{T}$ is connected. Thus if it includes any components not in $T$, at least one of these - necessarily dicritical - will meet $T$. This intersection of $T$ with $D$ is distinct from the one constructed in Lemma 4.4 since this dicritical precedes $E_{m}$ while the other follows it. Thus again we have a contradiction.

Now suppose that $Y_{t, r e d}$ is smooth and $C_{2}$ and $C_{3}$ fail. Since $Y_{t, r e d}$ is smooth it is irreducible and $\tilde{\Gamma}_{t, r e d}$ is smooth. Thus $N_{2}=0$ and $E_{t}$ is empty, so $N_{3}, N_{4}$ and $N_{5}$ all vanish. As we have just seen, since $C_{2}$ and $C_{3}$ fail, $N_{6}=0$. Now $N_{1}$ vanishes if $Y_{t}$ is reduced, and also if $Y_{t, r e d}$ is smooth elliptic, so in these cases we have $\chi\left(\Gamma_{t}\right)=\chi\left(\Gamma_{\text {ggen }}\right)$.

To complete the proof of Theorem 4.2, it remains to show that in the case of equality $Y_{t, r e d}$ is smooth, i.e. $E_{t}$ is empty. We will assume this false, and aim for a contradiction.

Lemma 4.6 Under the conditions of Lemma 4.5, $E_{t}=\emptyset$.

Proof Suppose not, then by Lemma 4.5, $Y_{t}$ is reduced. Choose a component $T$ of $E_{t}$ such that no exceptional curve in $E_{t}$ lies above the highest curve $V_{m}$ in $T$ (if there is more than one highest curve in $T$, then by Lemma 4.4, $\#(T \cap D) \geq 2$ ). Write $Y_{t}=T+W_{t}$.

Since (by Lemma 4.5) $T$ is convex, in the chain of blowings-up that produces $Y$, the components $V_{1}, \ldots, V_{m}$ of $T$ appear consecutively, so we have surfaces $X_{0}, \ldots, X_{m}$ say. There is just one base point $P$, say in $T$ in $X_{m}$, and it lies in $V_{m}$ (this follows since $\#(T \cap D)=1$ ). Resolving this till there is no base point on $V_{m}$ gives the chain $D_{1}, D_{2}, \cdots, D_{k}$ of curves constructed in the proof of Lemma 4.4 (all now dicritical since $V_{m}$ is the highest vertical curve). If $Z_{0}=X_{m}$, these appear in turn on surfaces $Z_{1}, \ldots, Z_{k}$. Any further blowing up to produce $Y$ takes place at points not on $T$ and produces further dicritical curves.

We will use the same letter to denote the image of a curve in $Y$ in any of these intermediate surfaces, but avoid ambiguity by writing $(A . B)_{Z}$ to denote the intersection number of $A$ and $B$ considered as curves in the surface $Z$. Also if a blow up at a point in one surface produces an exceptional curve in the next, we denote the point by the same letter as the curve, but in lower case, e.g. $\quad D_{i} \rightarrow d_{i}$, and then $m_{d_{i}}(A)$ denotes the multiplicity of the curve $A$ at the point $d_{i}$ in the lower surface. Recall from $\S 1.1$ that if $A, B$ are curves through a smooth point $e$ on a surface $X$, and blowing $e$ up produces an exceptional curve $E$ and strict transforms $A, B$ in $Z$, then $m_{e}(A)=(E . A)_{Z}$ and $(A . B)_{X}=$ $m_{e}(A) m_{e}(B)+(A \cdot B)_{Z}$. 
Blowing up $v_{1}$ produces a vertical curve. Hence the multiplicity at $v_{1}$ of the member $Y_{t}$ of the pencil strictly exceeds the multiplicity of $Y_{g g e n}$. We shall obtain a contradiction to this inequality.

We have $m_{d_{i}}\left(Y_{\text {ggen }}\right)-m_{d_{i}}\left(W_{t}\right)=\left(D_{i} .\left(Y_{\text {ggen }}-W_{t}\right)\right)_{Z_{i}}$. As $Y_{g g e n}$ and $Y_{t}$ are linearly equivalent, and $Y_{t}-W_{t}=T$, this equals $\left(D_{i} . T\right)_{Z_{i}}$. But the only component of $T$ meeting $D_{i}$ in $Z_{i}$ is $V_{m}$, and as $V_{m}$ is smooth in $Z_{i-1}$ this intersection number is 1 .

Since the only intersections of $V_{m}$ with $Y_{\text {ggen }}$ or $W_{t}$ in $Z_{i-1}$ occur at $d_{i}$, we have

$$
\left(V_{m} \cdot Y_{g g e n}-V_{m} \cdot W_{t}\right)_{Z_{i-1}}=m_{d_{i}}\left(Y_{g g e n}\right)-m_{d_{i}}\left(W_{t}\right)+\left(V_{m} \cdot Y_{g g e n}-V_{m} \cdot W_{t}\right)_{Z_{i}},
$$

so adding up,

$$
\left(V_{m} \cdot Y_{g g e n}-V_{m} \cdot W_{t}\right)_{Z_{0}}=k+\left(V_{m} \cdot Y_{g g e n}-V_{m} \cdot W_{t}\right)_{Z_{k}} .
$$

But in $Z_{k}, V_{m}$ is disjoint from $Y_{g g e n}$ and meets $W_{t}$ transversely in just one point. Thus the right hand side here is equal to $k-1$. The left hand side is equal (by the same argument) to $V_{m} \cdot\left(Y_{t}-W_{t}\right)=V_{m} \cdot T$. This is equal to $r-1$ where $r$ is the number of other components of $T$ meeting $V_{m}$. In particular, if $T$ has just one component $V_{m}$ we obtain $0=r=k$, contradicting the fact that there is at least one dicritical meeting $V_{m}$.

Until $V_{m}$ has been blown down, none of the curves not in $T$ meet $V_{m-1}$, so none of the corresponding infinitely near points are proximate to $v_{m-1}$. It follows from (2) that each component of $Y_{g g e n}$ and $W_{t}$ has the same multiplicity at $v_{m-1}$ as at $v_{m}$, and hence that

$$
m_{v_{m-1}}\left(Y_{g g e n}\right)-m_{v_{m-1}}\left(W_{t}\right)=m_{v_{m}}\left(Y_{g g e n}\right)-m_{v_{m}}\left(W_{t}\right)=k-1 \geq 0 .
$$

In $X_{m}=Z_{0}$ none of the components of $T$ other than $V_{m}$ meet any component of $Y_{\text {ggen }}$ or $W_{t}$ outside $T$. Thus any such component which passes through $v_{1}$ must also pass through $v_{m}$, and no further infinitely near point of either $Y_{g g e n}$ or $W_{t}$ is proximate to $v_{m-1}$. We may thus apply Lemma 1.1 to see that there are nonnegative numbers $a, b$, determined solely by the proximity relations, such that for any component $A$ of these curves through $v_{m}, m_{v_{1}}(A)=a m_{v_{m-1}}(A)+b m_{v_{m}}(A)$.

We apply this to $Y_{g g e n}$ on one hand and to $W_{t}$ on the other. It then follows from (19) that $m_{v_{1}}\left(Y_{g g e n}\right)-m_{v_{1}}\left(W_{t}\right)=(a+b)(k-1) \geq 0$. However, since in $X_{0}$ all components of $T$ have been blown down, $m_{v_{1}}\left(Y_{t}\right)=m_{v_{1}}\left(W_{t}\right)$. Hence we have $m_{v_{1}}\left(Y_{\text {ggen }}\right) \geq m_{v_{1}}\left(Y_{t}\right)$, contradicting the fact that $V_{1}$ is vertical. This contradiction establishes the result.

We have proved a little more than asserted in Theorem 4.2: we see from Lemma 4.5 that if equality holds and $Y_{t}=m C$ is non-reduced (so $C$ is smooth elliptic), and if also the pencil has base points and hence $Y$ contains dicritical curves, the degree of inseparability of each of these must be divisible by $m$. 


\section{References}

[1] Artin, E., Algebraic numbers and algebraic functions, Gordon \& Breach, 1967.

[2] Bloch, S.J., Cycles on arithmetic schemes and Euler characteristics of curves, pp. 421-450 in Algebraic Geometry Bowdoin 1985 (ed. Spencer J. Bloch et al.), Proc. Symp in Pure Math. 46 Part II, Amer. Math. Soc. 1987.

[3] Bombieri, E. and D. Mumford, Enriques' classification of surfaces in characteristic $p$ III, Invent. Math. 35 (1976) 197-232.

[4] Broughton, S.A., On the topology of polynomial hypersurfaces, pp 167-178 in Singularities (ed. P. Orlik), Proc. Symp in Pure Math 40(1), Amer. Math. Soc., 1983.

[5] Broughton, S.A., Milnor numbers and the topology of polynomial hypersurfaces, Invent. Math. 92 (1988) 217-241.

[6] Campillo, A., Algebroid curves in positive characteristic, Springer Lecture Notes in Math. 8131980.

[7] Deligne, P. et. al. Groupes de monodromie en géométrie algébrique, II, Séminaire de Géometrie Algébrique du Bois-Marie 1967-69 (SGA 7 II), Springer Lecture Notes in Math. 3401973.

[8] Deligne, P., Intersections sur les surfaces régulières, exposé X in [7].

[9] Deligne, P., Le formalisme des cycles évanescents, exposé XIII in [7].

[10] Deligne, P., La formule de Milnor, exposé XVI in [7].

[11] Dolgachev, I., On the purity of the degeneration loci of families of curves, Invent. Math. 8 (1969) 34-54.

[12] Dolgachev, I., The Euler characteristic of a family of algebraic varieties, Math. USSR Sbornik 18ii (1972) 303-319.

[13] Fulton, W., Intersection Theory, Springer-Verlag, 1984.

[14] Grothendieck, A., et al., Cohomologie $\ell$-adique et fonctions L, Séminaire de Géometrie Algébrique du Bois-Marie 1965-66 (SGA 5), Springer Lecture Notes in Math. 5891977.

[15] Grothendieck, A., Formule d'Euler-Poincaré en cohomologie étale, exposé X in $[14]$. 
[16] Hà Huy Vui and Lê Dũng Tràng, Sur la topologie des polynômes complexes, Acta Math. Vietnamica 9 (1984) 21-32.

[17] Hartshorne, R., Algebraic Geometry, Springer Graduate Text in Math. 52 1977.

[18] Iversen, B., Critical points of an algebraic function, Invent. Math. 12 (1971) 210-224.

[19] Kodaira, K., On compact analytic surfaces II, Ann. of Math. 77 (1963) 563626.

[20] Lê Dũng Tráng and Claude Weber, Equisingularité dans les pinceaux de germes de courbes planes et $C^{0}$-suffisance, l'Enseignement Math. 43 (1997) 355-380.

[21] Ogg, A.P., Cohomology of abelian varieties over function fields, Ann. of Math. 76 (1962) 185-212.

[22] Ogg, A.P., Elliptic curves and wild ramification, Amer. J. Math. 89 (1967) $1-21$.

[23] Raynaud, M., Caractéristique d'Euler-Poincaré d'un faisceau et cohomologie des variétés abéliennes, pp. 12-30 in Dix exposés sur la cohomologie des schémas, North-Holland, 1968.

[24] Šafarevič, I.R., Principal homogeneous spaces defined over a function field, Amer. Math. Soc. Transl. ser. 237 (1964) 85-114.

[25] Saito, T., Conductor, discriminant and the Noether formula of arithmetic surfaces, Duke Math. J., 57 (1988) 151-173.

[26] Serre, J.-P., Représentations des groupes finis, 2. ed., Hermann, 1971.

[27] Shimada, I., On supercuspidal families of curves on a surface in positive characteristic, Math. Ann. 292 (1992) 645-669.

[28] Sun, X., Ramifications on arithmetic schemes, J. reine angew. math 488 (1977) $37-54$.

[29] Tate, J., Genus change in inseparable extensions of base fields, Proc. Amer. Math. Soc. 3 (1952) 400-406.

[30] Zariski, O., The theorem of Bertini on the variable singular points of a linear system of varieties, Trans. Amer. Math. Soc. 56 (1944) 130-140. Reprinted on pp. 242-252 of Oscar Zariski: Collected Papers vol I (ed. H.Hironaka and D. Mumford) MIT Press, 1972. 